\title{
BAY 61-3606, CDKi, and Sodium Butyrate Treatments Modulate p53 Protein Level and Its Site-Specific Phosphorylation in Human Vestibular Schwannomas In Vitro
}

\author{
Rohan Mitra, ${ }^{1}$ Rohini Keshava, ${ }^{2}$ Mathivanan Jothi,, ${ }^{3}$ Vikas Vazhayil, \\ Indira Devi Bhagavatula, ${ }^{4}$ and Rajalakshmi Gope ${ }^{1}$ \\ ${ }^{1}$ Department of Human Genetics, NIMHANS, 2900 Hosur Road, Bangalore 560029, India \\ ${ }^{2}$ Department of Studies in Sericulture/Life Sciences, Jnana Bharathi Campus, Bangalore University, Bangalore 560056, India \\ ${ }^{3}$ Department of Biotechnology, Bharathiar University, Coimbatore 641046, India \\ ${ }^{4}$ Department of Neuro Surgery, NIMHANS, Bangalore 560029, India
}

Correspondence should be addressed to Rajalakshmi Gope; rlgope@gmail.com

Received 18 August 2014; Revised 24 September 2014; Accepted 7 October 2014; Published 20 October 2014

Academic Editor: Wenrui Duan

Copyright (C) 2014 Rohan Mitra et al. This is an open access article distributed under the Creative Commons Attribution License, which permits unrestricted use, distribution, and reproduction in any medium, provided the original work is properly cited.

\begin{abstract}
This study is done to evaluate the effect of spleen tyrosine kinase inhibitor (BAY 61-3606), cyclin-dependent kinase inhibitor (CDKi), and sodium butyrate $(\mathrm{Na}-\mathrm{Bu})$ on the level and phosphorylation of p53 protein and its binding to murine double minute 2 (MDM2) homologue in human vestibular schwannomas (VS). Primary cultures of the tumor tissues were treated individually with optimum concentrations of these small molecules in vitro. The results indicate modulation of p53 protein status and its binding ability to MDM2 in treated samples as compared to the untreated control. The three individual treatments reduced the level of total p53 protein. These treatments also decreased Ser392 and Ser15 phosphorylated p53 in tumor samples of young patients and Ser315 phosphorylated p53 in old patients. Basal level of Thr55 phosphorylated p53 protein was present in all VS samples and it remained unchanged after treatments. The p53 protein from untreated VS samples showed reduced affinity to MDM2 binding in vitro and it increased significantly after treatments. The MDM2/p53 ratio increased approximately 3 -fold in the treated VS tumor samples as compared to the control. The differential p53 protein phosphorylation status perhaps could play an important role in VS tumor cell death due to these treatments that we reported previously.
\end{abstract}

\section{Introduction}

The human vestibular schwannomas (VS) are benign tumors of the vestibular branch of the 8th cranial nerve. Bilateral VS tumors are mostly familial in nature, also known as neurofibromatosis type 2 (NF2) and as opposed to unilateral tumors which are generally sporadic. The onset age of familial forms of these tumors is earlier than that of the sporadic forms. These tumors cause high morbidity and also cause hearing loss, nausea, vertigo, tinnitus and facial paralysis, and hydrocephalus. Mortality rates are low due to this tumor. However, a higher rate of mortality is associated with secondary malignant tumors including gliomas and meningiomas $[1,2]$.

The $\mathrm{p} 53$ protein is an important cellular component with numerous key cellular functions $[3,4]$. One of its functions involves its ability to bind to DNA and regulate transcription and in turn regulate a variety of cellular processes such as cellcycle, apoptosis, DNA repair, aging, and senescence [5-8]. It also regulates a variety of cellular signals upon DNA damage, oncogenic activation, telomere erosion, change in cellular contact and adhesion, and hypoxia $[5,7,8]$. The p53 protein undergoes posttranslational modifications (PTMs) and one such important modification is phosphorylation [9-11]. It has an array of serine/threonine phosphorylation sites that spans the entire protein but the majority of them are found in the $\mathrm{N}$ and $\mathrm{C}$ terminal regulatory regions [12]. This protein is phosphorylated at 13 serine residues and 5 threonine residues. Ten of these phosphorylations occur in the amino terminal trans-activation domain, 1 occurs in the tetramerisation domain, 1 occurs in the proline-rich domain, 3 occur in the 
DNA binding domain, and 3 occur in the carboxy terminal regulatory domain [10]. The site-specific p53 phosphorylation is one of the important PTMs which affects its many functions including its function as a tumor suppressor [10, 13-17]. The current study is undertaken to evaluate the effect of small molecules on the p53 phosphorylation and its correlation to VS tumor cell survival in vitro.

Cyclin/CDK complexes have important role in cellular signaling as well as cell proliferation [18]. The spleen tyrosine kinase (Syk) is involved in the signaling pathways in hematopoietic cells and is suggested to be a part of signaling in nonhematopoietic cell types as well $[19,20]$. BAY 61-3606 is a known inhibitor of Syk and previous reports have shown its anti-inflammatory effects in vitro and in vivo [21]. $\mathrm{Na}-\mathrm{Bu}$ and other butyric acid derivatives have differentiating and antiproliferative effect in colonic epithelium [22, 23] and it is also a known inhibitor of histone deacetylase (HDAC) [24]. Kinases have important role in normal cell division and differentiation and altered kinase activities have been implicated in cancer development and metastasis. Therefore, kinase inhibitors are one of the important targets among cancer therapeutics $[25,26]$.

In this study we have analysed the effect of BAY 61-3606, $\mathrm{CDK}$ inhibitor (CDKi), and Na-Bu on the level and site-specific phosphorylation of $\mathrm{p} 53$ protein in human VS tumors in vitro. In addition, effect of these treatments on the binding affinity of MDM2 to the p53 protein was also analysed. The results were compared between the untreated controls and the treated ones. Our results show that modulation of p53 level, its site-specific phosphorylation, and its binding affinity to MDM2 by these inhibitors could affect VS tumor cell survival/death in vitro.

\section{Methods}

2.1. VS Tumor Tissue and Primary Culture. This study was approved by the National Institute of Mental Health and Neuro Sciences (NIMHANS, Bangalore, India) human ethics committee which is in accordance with Indian Council of Medical Research (ICMR, India) ethical guidelines for biomedical research on human subjects (2000). Informed consent was obtained from all patients prior to the collection of tissue samples. Only the patients with VS tumor were included in this study. Patients with other medical conditions such as diabetes and heart diseases and the patients who are taking prescription medications for other ailments were excluded from this study. A total of 20 fresh VS tumor tissues were collected from the NIMHANS Neurosurgery Department operation theater. Ten of these samples were from patients belonging to 35 years or younger and the remaining 10 were from older patients of above 35 years of age. The experiments were done in triplicate with each tumor tissue. The cell culture media, antibiotic, and the serum were obtained from Sigma-Aldrich, St. Louis, MO, USA. The primary VS tumor culture was established as described previously $[27,28]$. Briefly, the fresh VS tumor tissues were collected immediately after surgery, washed extensively in phosphate buffered saline (PBS), and minced into small pieces.
No collagenase or trypsin treatments which are generally employed to release loose cells from solid tissues were applied in our experiments as they are known to disrupt the VS tumor tissue or tumor cell integrity. Therefore, the VS tumor tissues used in this study retained most biological properties such as cell-cell contact, cell integrity, and to some extent tumor cell microenvironment as they existed in the patients in vivo. The tumor tissue pieces were placed in DMEM supplemented with $10 \%$ glucose. The DMEM also consisted of $10 \%$ fetal bovine serum (FBS), 100 units/mL penicillin and $100 \mu \mathrm{g} / \mathrm{mL}$ streptomycin. The tumor tissues were tweezed with forceps, minced with a sharp blade and loose tumor cells were separated during this process. These cells and tissues were then cultured in $35 \mathrm{~mm}$ Petri dishes (Sigma-Aldrich, St. Louis, MO) and incubated in a humidified atmosphere of 5\% $\mathrm{CO}_{2}$ and $95 \%$ air at $37^{\circ} \mathrm{C}$. The untreated control and $\mathrm{Na}-\mathrm{Bu}$, CDKi, and BAY 61-3606 treated samples were processed for Western blots. The time of addition of the small molecules was taken as "zero" hour. Two tumor samples were used for time-course experiment to standardize the optimum time point. One of these samples was from a young patient of 26 years and the other was from a 55-year-old patient. The same two tumor samples were also used for immunoprecipitation followed by Western blot analysis. A total of 20 tumor samples were treated for $48 \mathrm{~h}$ with the individual small molecules and used for Western blot analysis.

2.2. WI38 Cells. The normal human lung fibroblast cell line WI38 was obtained from the National Center for Cell Science (NCCS, Pune, India). This cell line was originally obtained from American Type Culture Collection (ATCC, USA). It is then distributed to other labs for research purpose only. The cells were propagated at $37^{\circ} \mathrm{C}$ with $5 \% \mathrm{CO}_{2}$ in a $95 \%$ humidified incubator as described previously $[13,14]$. The cells were fed with DMEM (Sigma-Aldrich, St. Louis, MO, USA) containing $10 \%$ fetal bovine serum (FBS) and propagated to approximately 70 to $80 \%$ confluency in $100 \mathrm{~mm}$ cell culture dishes in presence of penicillin $(100 \mathrm{U} / \mathrm{mL})$ and streptomycin $(100 \mu \mathrm{g} / \mathrm{mL})$. The plates containing the cells were washed thrice with phosphate buffered saline (PBS) and lysed with Tri reagent (Sigma-Aldrich, St. Louis, MO, USA). The protein was extracted according to the protocol supplied by Sigma-Aldrich and the concentration was estimated using spectrophotometer at $280 \mathrm{~nm}$. The protein lysate from the untreated VS tumor samples was used as internal controls. In addition, the WI38 protein was used as an additional control in the Western blot and immunoprecipitation analyses.

2.3. Na-Bu, CDKi, and BAY 61-3606 Treatments. Na-Bu, CDKi (2,4-diamino-5-oxo-pyrimidine hydrochloride), and BAY 61-3606 (2-[[7-(3,4-dimethoxyphenyl)imidazole[1,2-c] pyrimidin-5-yl] amino]pyridine-3-carboxamide hydrochloride) were obtained from Sigma-Aldrich, St. Louis, MO, USA. The stock solutions were prepared in water and the treatments were done as described previously [27, 28]. Briefly, twenty-four hours after seeding the VS tumor tissues to the Petri plates, one set of plates was left untreated and used as control, and the others were treated only once with $\mathrm{Na}-\mathrm{Bu}$, 
CDKi, or BAY 61-3606 at a final concentration of $2 \mathrm{mM}$ [22], $5 \mathrm{nM}$ [26], and $10 \mathrm{nM}$ [21], respectively. The small molecules solubilized in water were directly added to the Petri plates containing VS tumor tissues [27, 28]. For the time-course experiment two tumor samples, one from 26-year-old and the other from 55- year-old patients, were treated for 24, 48, and 72 hours and processed after treatment. However, the Western analysis for the time course experiment was done thrice; the average values were calculated and plotted in histograms.

2.4. Western Blotting. Qualitative and quantitative analysis of the $\mathrm{p} 53$ protein in the untreated and treated tumor samples were done by Western blotting as previously described [27, 28]. Briefly, the tumor samples were washed thrice in PBS, resuspended in $500 \mu \mathrm{L}$ of lysis buffer, and disrupted using Polytron tissue homogenizer (Kinematica AG, Switzerland), clarified by centrifugation, and the supernatant was transferred to a fresh Eppendorf tube. One hundred fifty micrograms of protein from each lysate was separated on $7.5 \%$ SDS-PAGE gels and transferred to PVDF membrane (SigmaAldrich, St. Louis, MO). A 9\% SDS-PAGE gel was used to detect the $11 \mathrm{kDa}$ S-100A2 protein [27, 28]. High-molecular weight prestained marker (broad range markers, sc-2361; Santa Cruz Biotechnology, Santa Cruz, CA, USA) was used as molecular weight standard. All the gels were run in duplicate, and one set was incubated with total p53 antibody or phospho-specific p53 antibodies. The other membrane was probed with $\gamma$-tubulin antibody (clone GTU-88, T5326; Sigma-Aldrich, St. Louis, MO, USA) which acted as an internal loading control. The primary antibody for p53 (sc$126,1: 500)$ and the phospho-specific p53 antibodies for Ser392 (sc-7997, 1:500), Ser315 (sc-17106-R, 1:500), Ser15 (sc-54277-R, 1:500), and Thr55 (sc-12904-R, 1:500) and the corresponding AP-conjugated secondary antibodies were purchased from Santa Cruz Biotechnology, Santa Cruz, CA, USA. These membranes were washed, incubated with appropriate secondary antibodies, and developed with one step NBT-BCIP (Sigma-Aldrich, St. Louis, MO, USA).

2.5. Immunoprecipitation. Immunoprecipitation protocol was as described previously $[16,29]$ and as prescribed by Santa Cruz Biotechnology, Santa Cruz, CA, USA. The MDM2 (WH0004193M1-100 UG) and $\gamma$-tubulin primary antibodies and the corresponding secondary antibodies were obtained from Sigma Aldrich, St. Louis, MO, USA. The protein A agarose (sc-2001) was obtained from Santa Cruz Biotechnology, Santa Cruz, CA, USA. Briefly, $200 \mu \mathrm{L}$ of tumor tissue lysates was incubated with $0.1 \mu \mathrm{g}$ of p53 primary antibody (mentioned under Western blot) for 2 hours at $4^{\circ} \mathrm{C}$. Ten microliters of protein A conjugated agarose was added to this mix and incubated on rocker at $4^{\circ} \mathrm{C}$ for an additional 2 hours. This mixture was centrifuged at $3000 \mathrm{rpm}$ for 30 seconds at $4^{\circ} \mathrm{C}$, washed twice in PBS, resuspended in $2 \mathrm{x}$ electrophoresis sample buffer, boiled and loaded on to SDS-PAGE gels, and processed as described under Western blot. The membranes were probed individually with either MDM2 or p53 antibody.
2.6. Specificity of Control and $p 53$ Antibodies. The phosphospecific antibodies are known to recognise the p53 protein only when the specific sites are phosphorylated and they do not cross react with unphosphorylated forms or other phosphorylated forms of p53 protein. The p53 antibody and the phospho-specific antibodies recognised $53 \mathrm{kDa}$ protein bands. The MDM2 antibody recognised a major $90 \mathrm{kDa}$ band and two minor bands one slightly above and the other below the major band. The $\gamma$-tubulin antibody recognised a $48 \mathrm{kDa}$ protein band.

2.7. Quantitation. The levels of protein bands were estimated by measuring the band intensities using Bio-Rad (Bio-Rad, Australia) quantity 1 software for all the samples individually, and these values were normalized to the corresponding control $48 \mathrm{kDa} \gamma$-tubulin bands from the same samples $[27,28]$. The levels of p53 protein in WI38 cell line were taken as 1.0 and the levels in untreated controls samples were calculated based on their intensities and reported as level of increase $[13,14]$. The protein levels in the treated samples were calculated for each tumor tissue based on the corresponding untreated control and reported as level of decrease in the treated samples $[13,14]$. Thus the p53 level in the WI38 cell line was used only to estimate the protein levels in untreated control samples and then the comparisons were done only between the untreated control and treated samples. The MDM2/p53 ratio for the WI38 cell line was taken as 1.0. The $\mathrm{p} 53$ and MDM 2 protein band intensities were measured from the treated and untreated samples and the ratios of MDM2/p53 were calculated using the ratio of WI38 as standard.

2.8. Statistical Analysis. Statistical analysis of the data was performed using Statistical Package for Social Sciences (SPSS) version 18.0. Paired $t$-test was performed for all the values. Generalized linear model repeated measures analysis of variance (RMANOVA) one-way classification was used. The overall levels of p53 protein and phosphorylated p53 proteins between the untreated control and $\mathrm{Na}-\mathrm{Bu}, \mathrm{CDKi}$, and BAY 61-3606 treated samples were analysed for statistical significance $[27,28]$. A $P$ value $<0.05$ was considered as statistically significant.

\section{Results}

3.1. Effect of $\mathrm{Na}-\mathrm{Bu}, \mathrm{CDKi}$, and BAY 61-3606 Treatments on the Level of 553 Protein. The untreated VS tumor sample had increased level of p53 protein as compared to the basal level found in WI38 cell line. There was an overall decrease in the level of $\mathrm{p} 53$ protein in the treated VS samples as compared to the untreated control. $\mathrm{Na}-\mathrm{Bu}, \mathrm{CDKi}$, and BAY 61-3606 treatments lead to a 2- to 3-fold decrease in the level of p53 protein as compared to the untreated control samples and it was statistically significant with a $P$ value $<0.05$. The decreased level of p53 protein was observed in all the VS tumor samples 48 hours after treatment (Figures 1(a) and 1(b)). 


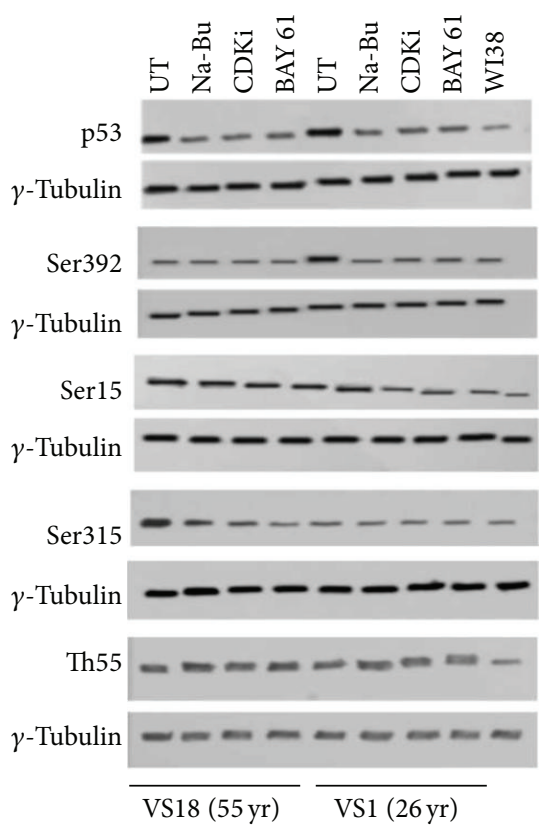

(a)

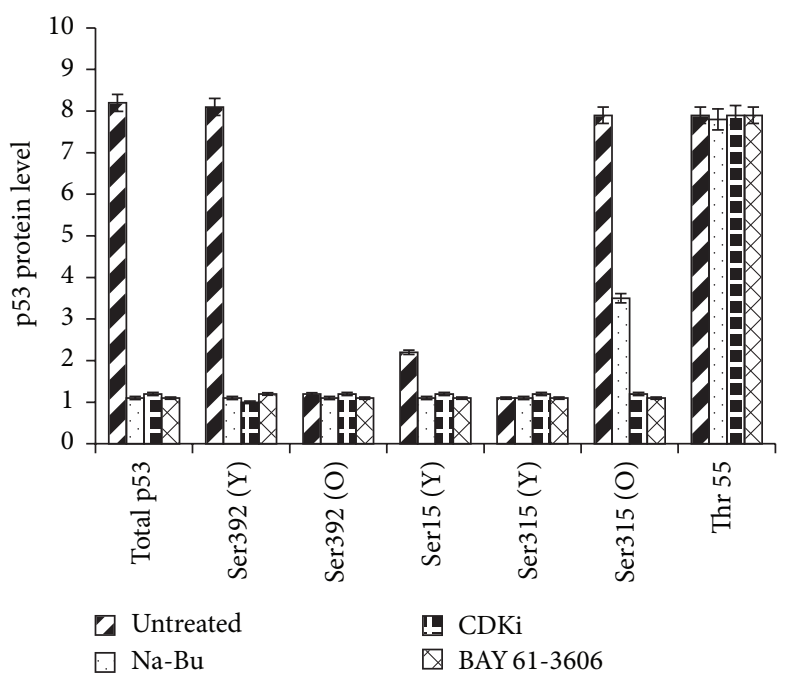

(b)

FIGURE 1: (a) Representative Western blot analysis of the effect of Na-Bu, CDKi, and BAY 61-3606 on the level of p53 protein in VS tumor sample in vitro 48 hours after treatment. The $53 \mathrm{kDa} 553$ band and the $48 \mathrm{kDa} \gamma$-tubulin bands are marked as indicated on the left. The WI38 was used as an additional control. VS18 is a tumor sample from a 55-year-old female patient; VS1 is a tumor sample from 26-year-old male patient. The lanes are as marked above: UT, untreated; Na-Bu, sodium butyrate treated; CDKi, CDKi treated; BAY 61, BAY 61-3606 treated. (b) Histogram of the combined data from VS tumor samples showing level of p53 and various phosphorylated forms of p53 proteins 48 hours after treatment. Total p53 and Thr55 bars represent data for 20 VS tumor samples. Ser392 (Y) and Ser392 (O) represent combined data from 10 young (35 years or younger) and 10 old (above 35 years old) patients, respectively. Ser315 (Y) and Ser315 (O) represent the same young and old patients as mentioned for Ser392. Ser15 (Y) data is from young patients only. The standard deviations are as indicated in the chart; $n=3$.

3.2. Effect of $\mathrm{Na}-\mathrm{Bu}, \mathrm{CDKi}$, and $\mathrm{BAY}$ 61-3606 Treatments on the Site-Specific Phosphorylation of p53 Protein. Higher levels of Ser392 phosphorylated p53 protein were found only in the VS tumor samples from young patients of 35 years or younger as compared to the normal WI38 cell line. Upon all the three treatments this level reduced by approximately 3fold to a basal level found in WI38 cell line. A basal level of Ser392 phosphorylated p53 protein was found in the old patients who were above 35 years of age and the basal level remained unaffected upon all the three treatments (Figures $1(a)$ and $1(b))$.

Relatively higher levels of Ser15 phosphorylated p53 protein were found in all the VS tumor samples as compared to the basal level found in WI38 cell line. The level of Ser15 p53 protein decreased by approximately 1.5 - to 2 -fold only in the VS tumor tissues of young patients upon all the three treatments. This decrease was parallel to the decrease observed for Ser392 p53 by the same treatments. The levels of Ser15 phosphorylated p53 protein remained unchanged by these treatments in the VS tumor samples from older patients who were above 35 years of age (Figures 1(a) and 1(b)).

Higher level of Ser315 phosphorylated form of $\mathrm{p} 53$ protein was found only in the VS tumor tissues of the patients who were above 35 years of age as compared to WI38 cell line and it decreased by 2.5 -fold upon $\mathrm{Na}$-Bu treatment. The CDKi and BAY 61-3602 treatments lead to more than 3-fold decrease in the level of Ser315 phosphorylated p53 protein. The VS tumor tissues from the young patients showed a basal level of Ser315 phosphorylated p53 protein, similar to WI38 cell line, and it remained unchanged upon all the three individual treatments (Figures 1(a) and 1(b)). The decreased level of phosphorylated p53 protein in the treated VS tumor samples as compared to the untreated ones was statistically significant with a $P$ value $>0.05$.

The levels of Thr55 phosphorylated p53 proteins were slightly higher in all the VS tumor samples as compared to the WI38 cell line and it remained almost unaffected by all the three individual treatments (Figures 1(a) and 1(b)).

3.3. Levels of $p 53$ and Phospho $p 53$ Proteins at Various Time Intervals after $\mathrm{Na}-\mathrm{Bu}, \mathrm{CDK} i$, and $\mathrm{BAY}$ 61-3606 Treatments. Results from the time-course experiment with VS tumor sample from young patient (VS4, 26 years) showed 1.5-fold decrease in p53 protein level with $\mathrm{Na}-\mathrm{Bu}$ treatment and 2fold decrease with CDKi or BAY 61-3606 treatments at 24 hours after treatment (Figures 2(a) and 2(c)). After 48 hours a 2.5- to 3 -fold decrease in the p53 protein level was found in the treated samples. In addition, 2- to 3-fold decrease in Ser392 (Figure 2(d)) and Ser15 (Figure 2(e)) phosphorylated p53 protein was found 24 hours after treatment for all the three treatments and it remained relatively unchanged 48 and 72 hours after treatments in this sample (VS4, 26 years). 


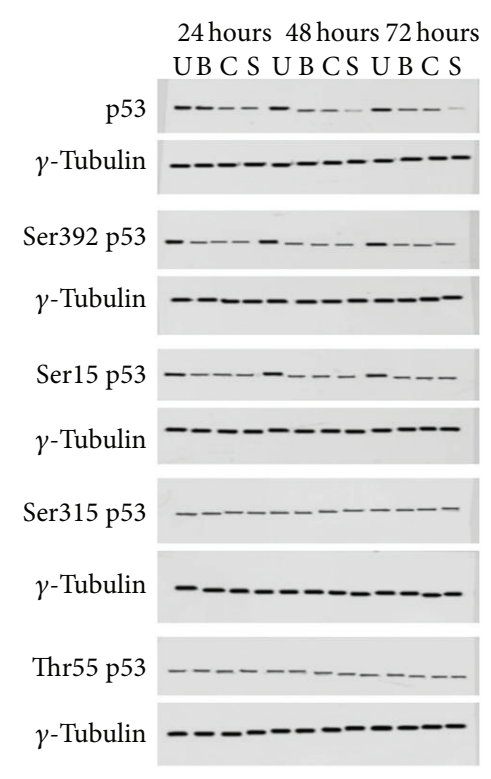

VS from young patient number 4 (26 years old)

(a)

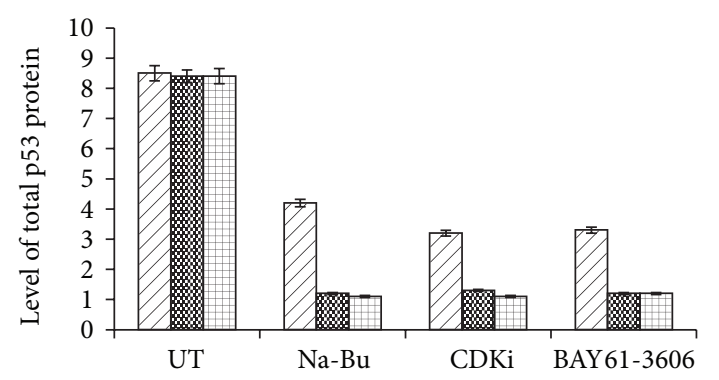

(c)

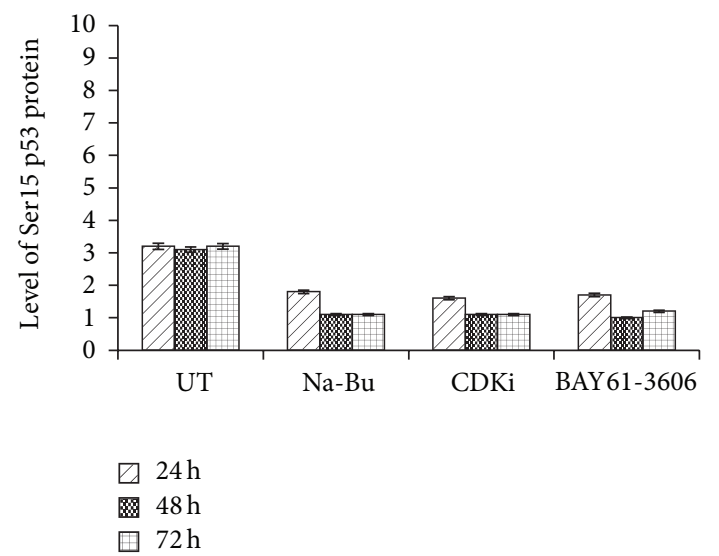

(e)

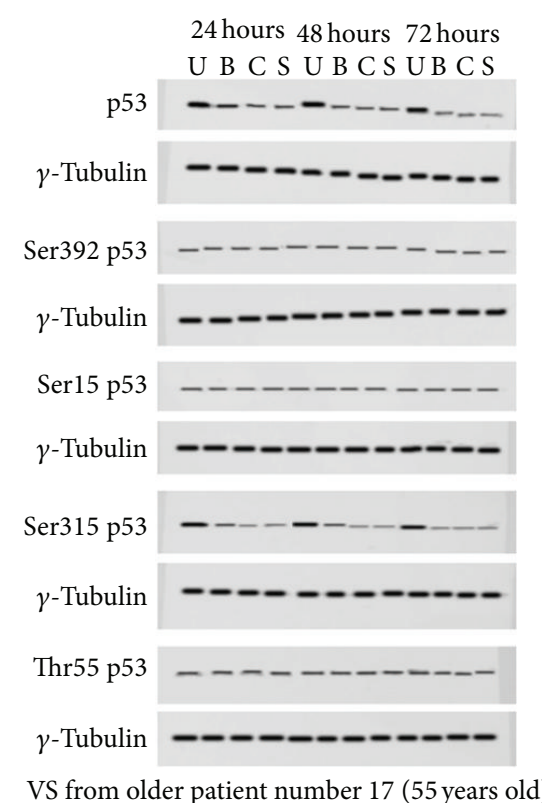

(b)

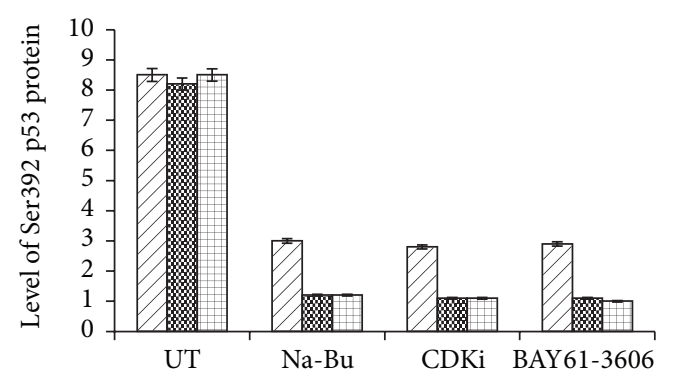

(d)

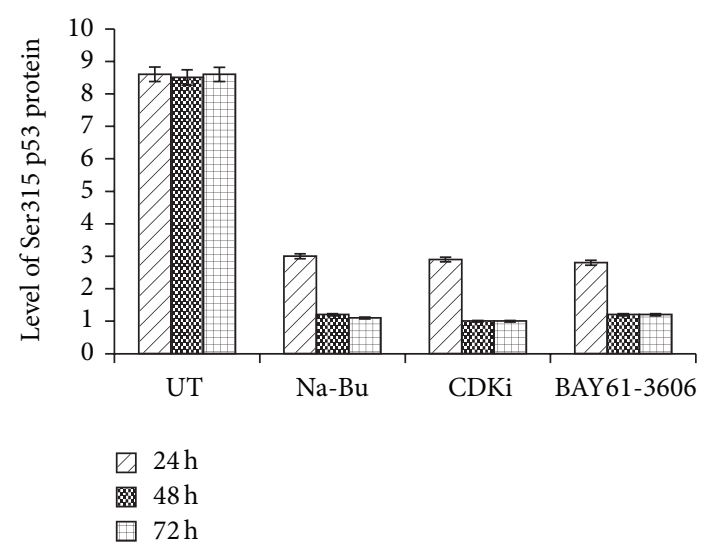

(f)

Figure 2: (a) Western blot showing level of total and phospho p53 proteins at 24, 48, and 72 hours after treatments. The sample used is from VS4 (26 years of age). U, untreated; B, Na-Bu; C, CDKi; S, BAY 61-3606, $n=3$. (b) Western blot analysis of level of total and phospho p53 proteins at 24, 48, and 72 hours after treatment. This data is from sample number VS17 (55 years of age). U, untreated, B, Na-Bu, C, CDKi, S, BAY 61-3606, $n=3$. (c) Histogram indicating level of total p53 protein at 24, 48, and 72 hours after treatment. This is data from sample VS4 (26 years) sample. UT, untreated; the treatments are as indicated in the figure, $n=3$. (d) Histogram indicating level of phospho Ser392 p53 protein after treatment at various time points. This data is from VS4 (26 years old) sample. UT, untreated; the treatments are as indicated. Basal level of Ser392 p53 protein was found in older patients of above 35 years of age and it remained unchanged with all the three treatments, $n=3$. (e) Level of Ser15 p53 protein at various time points after treatment. This data is from VS17 (26 years old). UT, untreated; other treatments are as shown in the figure. (f) Level of Ser315 protein after treatment at various time points. This data is from VS17 (55 years old). UT, untreated; the other treatments are shown in figure. Basal level of Ser315 p53 protein was found in young VS patients and the level remained unchanged upon all the three treatments. 


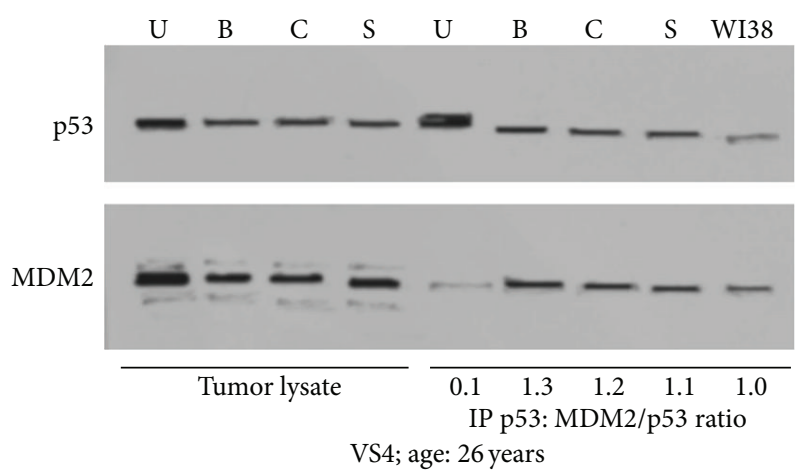

(a)

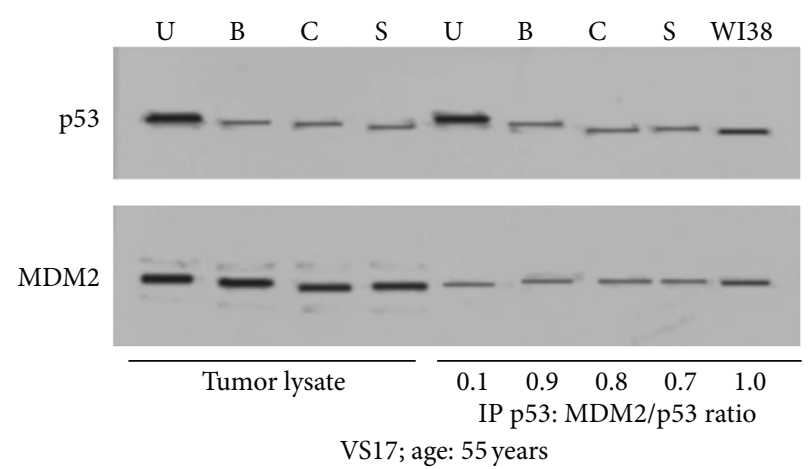

(b)

FIGURE 3: Western blot analysis of the effect of Na-Bu, CDKi, and BAY 61-3606 on the MDM2/p53 ratio in VS tumor sample 48 hours after treatment in vitro. The Western blot analysis was done after immunoprecipitation with $\mathrm{p} 53$ antibody as given under Methods. The $53 \mathrm{kDa}$ p53 band and the $90 \mathrm{kDa}$ MDM2 bands are as given on the left. The levels of p53 and MDM2 in the tumor lysates are in the left panel. The $\mathrm{MDM} 2 / \mathrm{p} 53$ ratios after immunoprecipitation are given on the right panel and the actual ratios are given below the corresponding lanes. The MDM2/p53 ratio of WI38 was taken as 1.0 and used as a control. (a) shows data for VS sample from young patient (26 years old); (b) shows data for VS sample from old patient (55 years old). Lane U, untreated; Lane B, Na-Bu treated; Lane C, CDKi treated; Lane S, BAY 61-3606 treated.

A basal level of Ser315 phosphorylated p53 protein was found in VS tumor of young patient (VS4, 26 years) and it remained unchanged with the treatments at various time points (Figure 2(a)).

Results from the time-course experiment with VS tumor sample from old patient of 55 years of age (VS17) showed a 2.5 - and 3 -fold decrease in p53 protein level 24 hours and 48 hours, respectively, after $\mathrm{Na}-\mathrm{Bu}$ treatment and little change was observed between 48 and 72 hours after treatment (Figure 2(c)). The Na-Bu treatment led to 1.5- and 2-fold decrease of Ser $315 \mathrm{p} 53$ protein at 24 and 48 hours, respectively. A 2- to 3-fold decrease of Ser315 was observed with CDKi and BAY 61-3606 treatments and it was found early as 24 hours after treatment (Figures 2(b) and 2(f)). The levels of Ser392 and Thr55 phosphorylated p53 protein from the VS tumor sample of this old patient remained unchanged after treatments (Figure 2(b)). The level of Ser15 decreased slightly upon treatments at various time intervals and it was also observed in samples from young patients (Figures 2(a) and $2(\mathrm{e}))$.

3.4. Effect of $\mathrm{Na}-\mathrm{Bu}, \mathrm{CDKi}$, and $B A Y$ 61-3606 on the MDM2 Binding to 553 Protein in VS Tumor Sample. Data from the immunoprecipitation studies showed that only a trace amount of MDM2 was bound to $\mathrm{p} 53$ protein in the VS tumor samples regardless of the age of the patients. Upon all the three individual treatments there was a 3 - to 3.5-fold increase in the ratio of MDM2/p53. This ratio was approximately 0.5 fold higher in the tumor tissue of young patient of 26 years of age as compared to that of the old patient who was 55 years of age. The normal human lung fibroblast cell line WI38 showed MDM2/p53 ratio of 1 (Figure 3).

\section{Discussion}

The VS tumors were confirmed by routine methods including histopathology, MRI scan, and presence of S-100A2 marker antigen [27, 28]. Optimum concentrations of inhibitors (1, 2 , and $5 \mathrm{mM}$ for $\mathrm{Na}-\mathrm{Bu} ; 0.5,1.0$, and $5.0 \mathrm{nM}$ for $\mathrm{CDKi} ; 2,5$, and $10 \mathrm{nM}$ for BAY 61-3606) and time-dependent (24, 48, and 72 hours) expression of caspase- 3 and PARP-1 cleavage were previously analyzed and published $[27,28]$.

Presence of higher levels of $\mathrm{p} 53$ protein observed in this study (Figures 1(a) and 1(b)) confirms our previous data on 2to 5- and 2- to 4-fold increase in the level of p53 mRNA and protein, respectively, in human VS tumors [13]. Previously we have also reported that the levels of p53 mRNA and protein in the normal human 8th cranial nerves obtained from autopsy cases were slightly less than that of the WI38 cell line [13]. Therefore, use of the p53 protein level in WI38 to calculate the level in the untreated VS tumor samples is acceptable in our experimental conditions [13] and the level of p53 protein in the WI38 cell line was used only to estimate the p53 levels in untreated control samples. Further comparisons were done between the untreated VS controls and the corresponding treated VS tumor samples. p53 gene mutation is not reported in human VS tumors and it was suggested that the tumor suppressor function of the wild type p53 protein perhaps is abrogated by other means such as modulation of its level and its site specific phosphorylation [13]. All the VS tumor samples used in the present study had no p53 gene mutation $[13,27]$.

The current data corroborate our previous report on agedependent Ser392 p53 phosphorylation in the VS tumors of young patients only (Figure 2(a)) [13]. Presence of Ser392 p53 protein is indicative of advanced tumor stage and grade and poor prognosis and large tumor size $[14,15,30,31]$. The MDM2 protein binds to $\mathrm{p} 53$, inhibits its function, and causes its degradation [32-34]. The Ser392 mutants have been reported to have reduced binding affinity to MDM2 [16]. Increased level of p53 protein in our previous report [13] and in the present study (Figure 1(b)) is due to its decreased binding affinity to MDM2 [16]. Conversely, the decreased p53 protein level in the treated cells observed in this study is due 
to its increased binding affinity to MDM2 and consequent MDM2-mediated degradation (Figure 3).

Ser392 phosphorylation status was shown to influence the phosphorylation at Ser15 [16] and our data are in agreement with this report (Figures 2(c), 2(d), 2(e), and 2(f)). Ser15 phosphorylation reduced the specific activity of $\mathrm{p} 53$ protein as a transcription factor in vivo [17]. In addition, this modification also reduced the binding affinity of $\mathrm{p} 53$ protein to its primary negative regulator HDM2 [12, 16, 35].

This is the first report showing the presence of increased level of Ser315 phosphorylation in the VS tumor samples from old patients of 35 years and above (Figures 2(e) and 2(f)). The phosphorylation of p53 protein at Ser392 and Ser315 sites are mutually exclusive $[36,37]$ and our data is in agreement with these reports [36, 37] (Figures 1(a) and 1(b)). Ser315 phosphorylation increases p53 transactivation potentially through nuclear retention and promotes its MDM2 dependent proteolysis [17, 38, 39]. The observed 3fold increase in the p53/MDM2 binding affinity in the treated VS sample from old patient supports these notions (Figure 3).

Thr55 p53 is constitutively phosphorylated in unstressed cells and dephosphorylated following stress $[9,40]$. Our data shows that the Thr55 p53 levels did not decrease upon treatments which indicates that the treated VS tumor cells perhaps are not reverting back to unstressed or normal state (Figures $1(\mathrm{a})$ and $1(\mathrm{~b})$ ).

The function of $\mathrm{p} 53$ in vivo can be determined by a variety of modifications at other phosphorylation sites in combination with modifications other than phosphorylation which can interpret multipotency of individual modifications [10, 41]. Therefore, it is important to explore the significance of combinations of p53 phosphorylations and perhaps other modifications in the VS tumor cell death that we reported previously $[27,28]$. It is important to note that all the three treatments also caused upregulation of cell death proteins (Shh, caspase-3, and cleaved PARP) and downregulation of key cell survival proteins (EGF-R, PI3K, NF- $\kappa$ B, and phospho pRb) $[27,28]$.

The observed cell death in the treated VS tumor samples appears to be via activated JNK and modulation of phosphorylation of p53 (this study) and pRb that we reported previously [27]. In addition, other components of the p53 mediated apoptotic pathway such as increased levels of p21, caspase 3 and cleaved PARP proteins as well as DNA fragmentation were found only in the treated samples [27, 28]. Na-Bu induces p53 independent Fas-mediated apoptosis in cultured MCF-7 human breast cancer cells [42]. However, the VS tumor cells are known to undergo apoptosis that is not Fas mediated as they lack Fas expression [43]. We found no detectable Fas protein in 17 out of 21 VS tumor samples (unpublished data) which is in agreement with the previously published report by Mawrin et al. [43]. The remaining three VS tumor samples had negligible level of Fas protein and it remained unchanged upon $\mathrm{Na}-\mathrm{Bu}$ treatment (unpublished data). Therefore, the Na-Bu induced VS tumor cell death observed in the present study appears to be not Fas mediated which is in agreement with previous report [43]. The p53 protein with decreased phosphorylation in the treated VS tumor cells could have triggered the apoptotic pathway before it was bound to and degraded by MDM2. Many proapoptotic indicators including increased levels of caspase-3 and p21 proteins and cleaved PARP-1 were found as early as 24 hours after treatment [28].

Wild-type p53 is known to take mutant like conformation and it was suggested that the function of p53 may be regulated via its ability to adopt distinct conformations [44]. It was also suggested that phosphorylation can alter the p53 conformation and thereby its function $[45,46]$. Therefore, it is important to assess the wild-type p53 protein with altered conformation as a drug target. It has been suggested that novel drugs should be formulated in order to prevent tumor development elicited by wild-type p53 protein with altered conformation [47]. Such drugs could also be used to manage tumors that have miss-folded wild-type p53 protein with altered PTMs. Thus, it is important to assess the posttranslational modifications (PTMs) of p53 protein especially in tumors harbouring wild-type 533 as these PTMs can alter the p53 protein conformation and thereby its function as a tumor suppressor.

$\mathrm{Na}-\mathrm{Bu}$ is a naturally occurring, nontoxic substance. Use of $\mathrm{Na}-\mathrm{Bu}$ as an anticancer agent has been proposed for various tumor types [22, 24]. BAY 61-3606 [21], an anti-inflammatory agent, is being evaluated as a cancer therapeutic considering cancer as an inflammatory disease. CDKi and its modified forms are also being developed as a potential cancer therapeutic and one such modified version was evaluated in the cancer clinical trials [26]. Therefore, we believe that the small molecules used in this study can be developed into possible therapeutics for managing human VS tumors. Data from the present study shows that the p53 protein level, its sitespecific phosphorylation, and its binding affinity to MDM2 are modulated in the small molecules treated VS tumor cells in vitro and it appears that these modulations could have a role on VS tumor cell death caused by these molecules $[27,28]$.

\section{Overall Conclusion}

Human VS tumors cause high morbidity. $\mathrm{Na}-\mathrm{Bu}$, modified CDK inhibitors, and BAY 61-3606 are being evaluated for their therapeutic potential in many human cancers. Our data shows that these small molecules affect the level and site-specific phosphorylation of $\mathrm{p} 53$ protein in vitro which could have caused the observed cell death in vitro. It would be interesting to evaluate the beneficial effects of these small molecules when combined with surgical resection or stereotactic radiosurgery which are currently used to manage human VS tumors.

\section{Ethical Approval}

This study was approved by NIMHANS Human Ethics Committee, clearance reference numbers: IEC no. RPA/105/07, dated 26 December, 2007, and no. NIMH/67th IEC/2009, dated 25 September, 2009. 


\section{Conflict of Interests}

The authors declare that there is no conflict of interests regarding the publication of this paper.

\section{Acknowledgments}

The authors wish to thank Dr. S. Sampath, Dr. D. P. Shukla, Dr. S. Dwarkanath, Dr. D. I. Bhat, Dr. N. Rao, Dr. M. Ranjan, and others including the residents of the NIMHANS Neuro Surgery Department who provided the tumor tissues used in this study. They thank Dr. D. K. Subbakrishna for his help in statistical analysis. They thank NIMHANS, for providing financial assistance for infrastructure for this study. Rohan Mitra is a Senior Research Fellow (SRF) supported by the DST project funds.

\section{References}

[1] R. L. Martuza and R. Eldridge, "Neurofibromatosis 2. (Bilateral acoustic neurofibromatosis)," The New England Journal of Medicine, vol. 318, no. 11, pp. 684-688, 1988.

[2] D. E. Brackmann and J. A. Kwartler, "A review of acoustic tumors: 1983-1988," American Journal of Otology, vol. 11, no. 3, pp. 216-232, 1990.

[3] D. Eliyahu, D. Michalovitz, S. Eliyahu, O. Pinhasi-Kimhi, and M. Oren, "Wild-type p53 can inhibit oncogene-mediated focus formation," Proceedings of the National Academy of Sciences of the United States of America, vol. 86, no. 22, pp. 8763-8767, 1989.

[4] C. A. Finlay, P. W. Hinds, and A. J. Levine, "The p53 protooncogene can act as a suppressor of transformation," Cell, vol. 57, no. 7, pp. 1083-1093, 1989.

[5] D. P. Lane, "Cancer: p53, guardian of the genome," Nature, vol. 358, no. 6381, pp. 15-16, 1992.

[6] D. Hanahan and R. A. Weinberg, "The hallmarks of cancer," Cell, vol. 100, no. 1, pp. 57-70, 2000.

[7] D. P. Lane and L. V. Crawford, "T antigen is bound to a host protein in SV40 transformed cells," Nature, vol. 278, no. 5701, pp. 261-263, 1979.

[8] H. F. Horn and K. H. Vousden, "Coping with stress: multiple ways to activate p53," Oncogene, vol. 26, no. 9, pp. 1306-1316, 2007.

[9] A. M. Bode and Z. Dong, "Post-translational modification of p53 in tumorigenesis," Nature Reviews Cancer, vol. 4, no. 10, pp. 793-805, 2004.

[10] B. Gu and W.-G. Zhu, "Surf the post-translational modification network of p53 regulation," International Journal of Biological Sciences, vol. 8, no. 5, pp. 672-684, 2012.

[11] B. Vogelstein, D. Lane, and A. J. Levine, "Surfing the p53 network," Nature, vol. 408, no. 6810, pp. 307-310, 2000.

[12] C. Dai and W. Gu, "P53 post-translational modification: deregulated in tumorigenesis," Trends in Molecular Medicine, vol. 16, no. 11, pp. 528-536, 2010.

[13] A. H. P. P. Dayalan, M. Jothi, R. Keshava et al., "Age dependent phosphorylation and deregulation of p53 in human vestibular schwannomas," Molecular Carcinogenesis, vol. 45, no. 1, pp. 3846, 2006.

[14] K. Rohini, J. Mathivanan, P. D. A. H. Prabhu et al., "Loss of heterozygosity of the p53 gene and deregulated expression of its mRNA and protein in human brain tumors," Molecular and Cellular Biochemistry, vol. 300, no. 1-2, pp. 101-111, 2007.
[15] J. K. Bar, I. Saomska, J. Rabczyńki, L. Noga, and M. Grybov, "Expression of p53 protein phosphorylated at serine 20 and serine 392 in malignant and benign ovarian neoplasms correlation with clinicopathological parameters of tumors," International Journal of Gynecological Cancer, vol. 19, no. 8, pp. 1322-1328, 2009.

[16] S. Piccinin, E. Tonin, S. Sessa et al., "A “twist box" code of p53 inactivation: twist box: p53 interaction promotes p53 degradation," Cancer Cell, vol. 22, no. 3, pp. 404-415, 2012.

[17] J. P. Blaydes, M. G. Luciani, S. Pospisilova, H. M.-L. Ball, B. Vojtesek, and T. R. Hupp, "Stoichiometric phosphorylation of human p53 at Ser315 stimulates p53-dependent transcription," The Journal of Biological Chemistry, vol. 276, no. 7, pp. 46994708, 2001.

[18] M. Malumbres and M. Barbacid, "Cell cycle, CDKs and cancer: a changing paradigm," Nature Reviews Cancer, vol. 9, no. 3, pp. 153-166, 2009.

[19] K. Sada, T. Takano, S. Yanagi, and H. Yamamura, "Structure and function of syk protein-tyrosine kinase," The Journal of Biochemistry, vol. 130, no. 2, pp. 177-186, 2001.

[20] S. Yanagi, R. Inatome, T. Takano, and H. Yamamura, "Syk expression and novel function in a wide variety of tissues," Biochemical and Biophysical Research Communications, vol. 288, no. 3, pp. 495-498, 2001.

[21] N. Yamamoto, K. Takeshita, M. Shichijo et al., "The orally available spleen tyrosine kinase inhibitor 2-[7-(3,4-dimethoxyphenyl)-imidazo[1,2-c]pyrimidin-5-ylamino]-nicotinamide dihydrochloride (BAY 61-3606) blocks antigen-induced airway inflammation in rodents," The Journal of Pharmacology and Experimental Therapeutics, vol. 306, no. 3, pp. 1174-1181, 2003.

[22] R. Gope and M. L. Gope, "Effect of sodium butyrate on the expression of retinoblastoma (RB1) and P53 gene and phosphorylation of retinoblastoma protein in human colon tumor cell line HT29," Cellular and Molecular Biology, vol. 39, no. 6, pp. 589-597, 1993.

[23] A. Rephaeli, E. Rabizadeh, A. Aviram, M. Shaklai, M. Ruse, and A. Nudelman, "Derivatives of butyric acid as potential antineoplastic agents," International Journal of Cancer, vol. 49, no. 1, pp. 66-72, 1991.

[24] J. R. Davie, "Inhibition of histone deacetylase activity by butyrate," The Journal of Nutrition, vol. 133, no. 7, pp. 2485S-2493S, 2003.

[25] D. Hanahan and R. A. Weinberg, "Hallmarks of cancer: the next generation," Cell, vol. 144, no. 5, pp. 646-674, 2011.

[26] W. DePinto, X.-J. Chu, M. Smith et al., "In vitro and in vivo activity of R547: a potent selective cyclin-dependent kinase inhibitor currently in phase I clinical trials," Molecular Cancer Therapeutics, vol. 5, no. 11, pp. 2644-2658, 2006.

[27] R. Mitra, B. I. Devi, M. L. Gope, D. K. Subbakrishna, and R. Gope, "Sodium butyrate modulates $\mathrm{pRb}$ phosphorylation and induces cell death in human vestibular schwannomas in vitro," Indian Journal of Experimental Biology, vol. 50, no. 1, pp. 19-27, 2012.

[28] R. Mitra, I. D. Bhagavatula, and R. Gope, "BAY 61-3606, CDKi, and sodium butyrate treatments alter gene expression in human vestibular schwannomas and cause cell death in vitro," ecancermedicalscience, vol. 6, no. 1, article 285, 2012.

[29] R. Gope and M. L. Gope, "Abundance and state of phosphorylation of the retinoblastoma susceptibility gene product in human colon cancer," Molecular and Cellular Biochemistry, vol. 110, no. 2, pp. 123-133, 1992. 
[30] M. Matsumoto, M. Furihata, A. Kurabayashi, and Y. Ohtsuki, "Phosphorylation state of tumor-suppressor gene $\mathrm{p} 53$ product overexpressed in skin tumors," Oncology Reports, vol. 12, no. 5, pp. 1039-1043, 2004.

[31] M. Matsumoto, M. Furihata, A. Kurabayashi et al., "Prognostic signifi cance of serine 392 phosphorylation in overexpressed p53 protein in human esophageal squamous cell carcinoma," Oncology, vol. 67, no. 2, pp. 143-150, 2004.

[32] Y. Haupt, R. Maya, A. Kazaz, and M. Oren, "Mdm2 promotes the rapid degradation of p53," Nature, vol. 387, no. 6630, pp. 296299, 1997.

[33] M. H. G. Kubbutat, S. N. Jones, and K. H. Vousden, "Regulation of p53 stability by Mdm2," Nature, vol. 387, no. 6630, pp. 299303, 1997.

[34] J. Momand, G. P. Zambetti, D. C. Olson, D. George, and A. J. Levine, "The mdm-2 oncogene product forms a complex with the $\mathrm{p} 53$ protein and inhibits $\mathrm{p} 53$-mediated transactivation," Cell, vol. 69, no. 7, pp. 1237-1245, 1992.

[35] F. Toledo and G. M. Wahl, "Regulating the p53 pathway: in vitro hypotheses, in vivo veritas," Nature Reviews Cancer, vol. 6, no. 12, pp. 909-923, 2006.

[36] J. Huang, L. Perez-Burgos, B. J. Placek et al., "Repression of p53 activity by Smyd2-mediated methylation," Nature, vol. 444, no. 7119, pp. 629-632, 2006.

[37] K. Sakaguchi, H. Sakamoto, M. S. Lewis et al., "Phosphorylation of serine 392 stabilizes the tetramer formation of tumor suppressor protein p53," Biochemistry, vol. 36, no. 33, pp. 1011710124, 1997.

[38] V. Fogal, J.-K. Hsieh, C. Royer, S. Zhong, and X. Lu, "Cell cycledependent nuclear retention of $\mathrm{p} 53$ by E2F1 requires phosphorylation of p53 at Ser315," The EMBO Journal, vol. 24, no. 15, pp. 2768-2782, 2005.

[39] H. Katayama, K. Sasai, H. Kawai et al., "Phosphorylation by aurora kinase A induces Mdm2-mediated destabilization and inhibition of p53," Nature Genetics, vol. 36, no. 1, pp. 55-62, 2004.

[40] H.-H. Li, X. Cai, G. P. Shouse, L. G. Piluso, and X. Liu, "A specific PP2A regulatory subunit, B56 $\gamma$, mediates DNA damageinduced dephosphorylation of p53 at Thr55," The EMBO Journal, vol. 26, no. 2, pp. 402-411, 2007.

[41] S. Saito, H. Yamaguchi, Y. Higashimoto et al., "Phosphorylation site interdependence of human $\mathrm{p} 53$ post-translational modifications in response to stress," The Journal of Biological Chemistry, vol. 278, no. 39, pp. 37536-37544, 2003.

[42] V. Chopin, R.-A. Toillon, N. Jouy, and X. Le Bourhis, "Sodium butyrate induces P53-independent, Fas-mediated apoptosis in MCF-7 human breast cancer cells," British Journal of Pharmacology, vol. 135, no. 1, pp. 79-86, 2002.

[43] C. Mawrin, E. Kirches, K. Dietzmann, A. Roessner, and C. Boltze, "Expression pattern of apoptotic markers in vestibular schwannomas," Pathology Research and Practice, vol. 198, no. 12, pp. 813-819, 2002.

[44] T. D. Halazonetis, L. J. Davis, and A. N. Kandil, "Wild-type p53 adopts a "mutant"-like conformation when bound to DNA," The EMBO Journal, vol. 12, no. 3, pp. 1021-1028, 1993.

[45] T. Minamoto, T. Buschmann, H. Habelhah et al., "Distinct pattern of p53 phosphorylation in human tumors," Oncogene, vol. 20, no. 26, pp. 3341-3347, 2001.

[46] Y. Peng, C. Li, L. Chen, S. Sebti, and J. Chen, "Rescue of mutant p53 transcription function by ellipticine," Oncogene, vol. 22, no. 29, pp. 4478-4487, 2003.
[47] D. Ishimaru, L. F. Maia, L. M. Maiolino et al., "Conversion of wild-type $\mathrm{p} 53$ core domain into a conformation that mimics a hot-spot mutant," Journal of Molecular Biology, vol. 333, no. 2, pp. 443-451, 2003. 


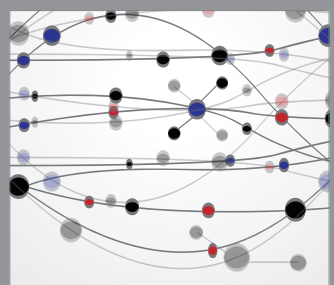

The Scientific World Journal
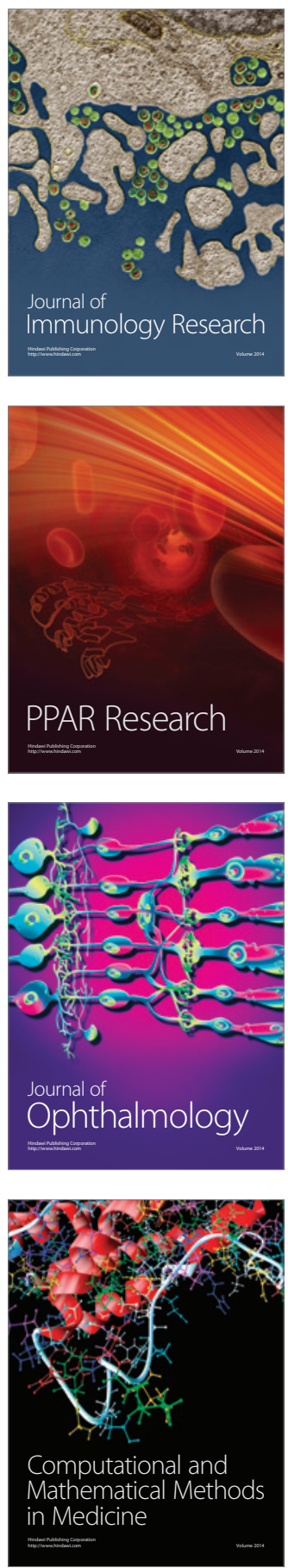

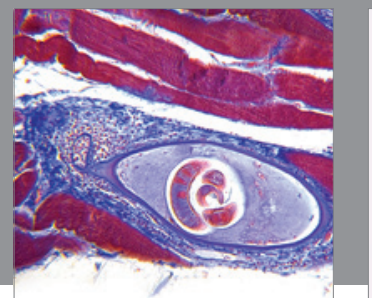

Gastroenterology

Research and Practice
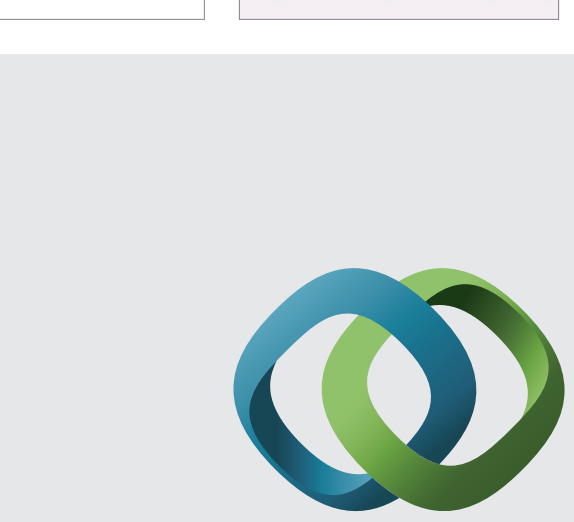

\section{Hindawi}

Submit your manuscripts at

http://www.hindawi.com
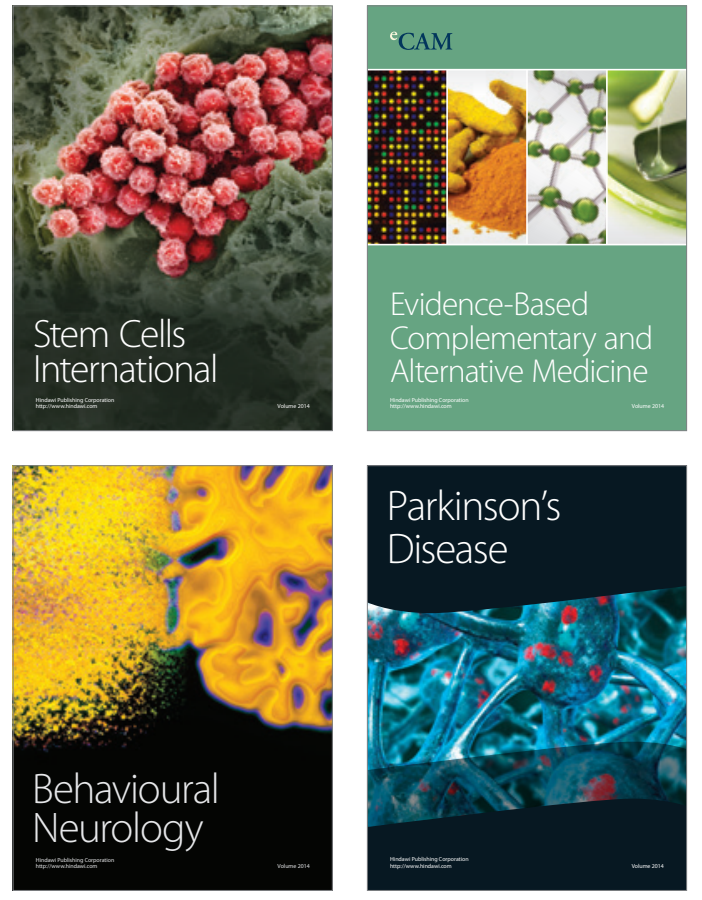
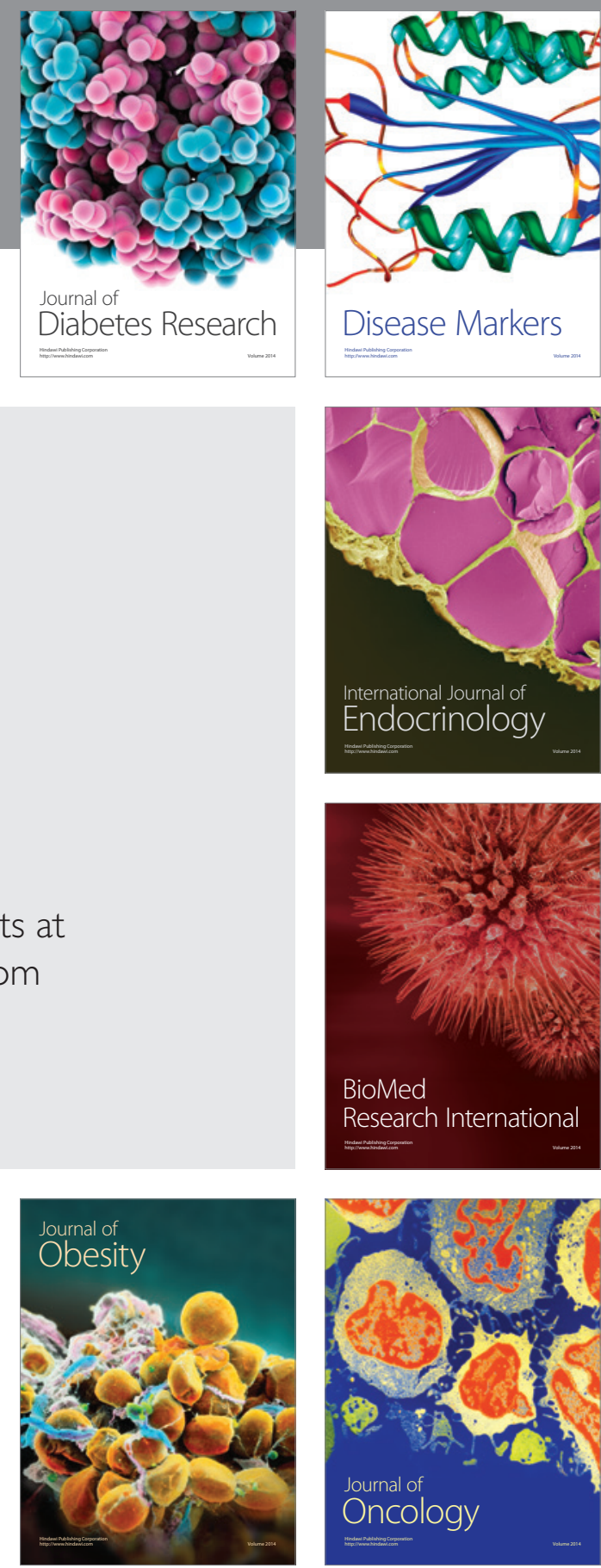

Disease Markers
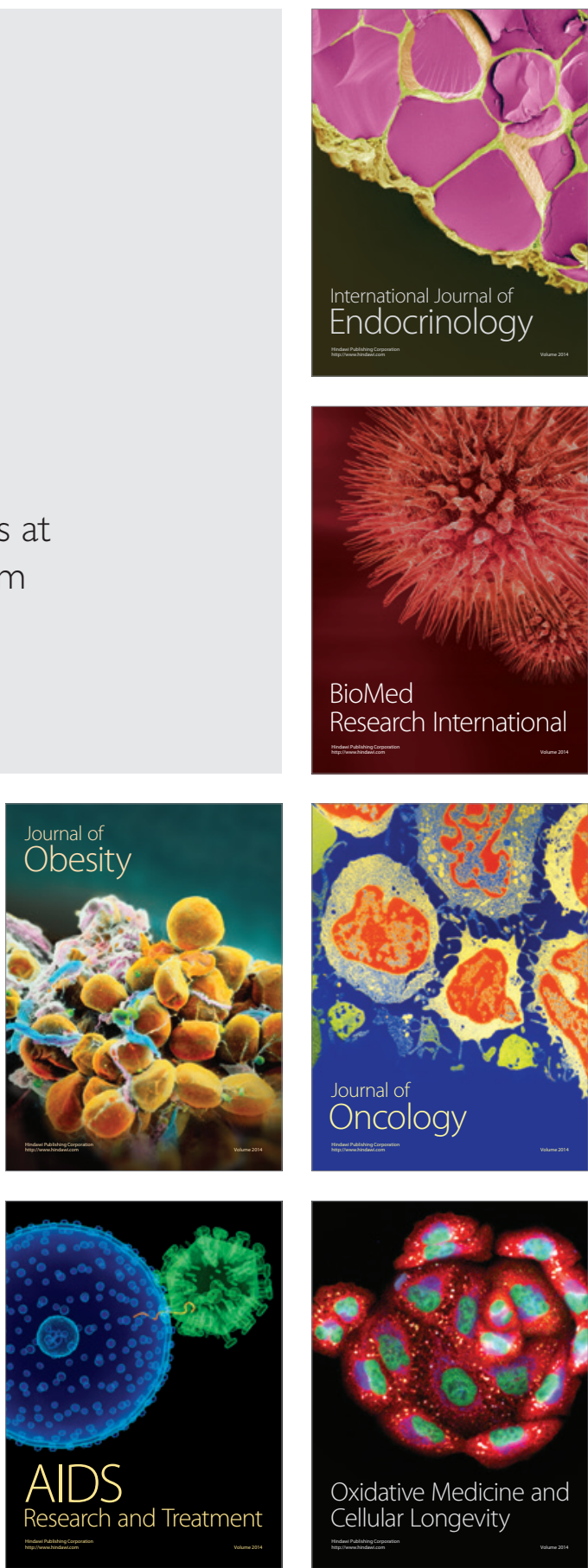